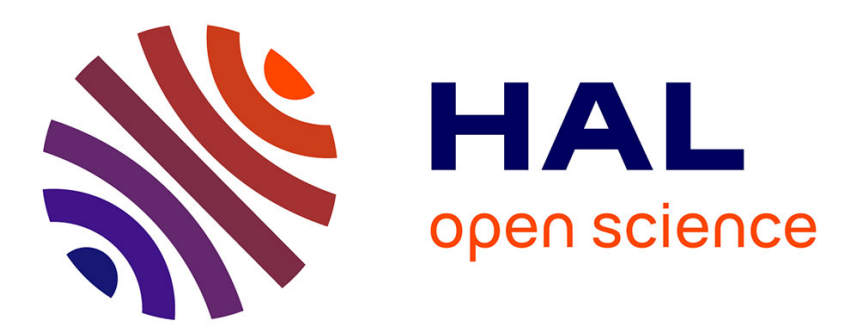

\title{
A numerical approach for the study of glass furnace regenerators
}

Y. Reboussin, Jean-François Fourmigue, Philippe Marty, Olivier Citti

\section{To cite this version:}

Y. Reboussin, Jean-François Fourmigue, Philippe Marty, Olivier Citti. A numerical approach for the study of glass furnace regenerators. Applied Thermal Engineering, 2005, 25, pp.2299-2320. 10.1016/j.applthermaleng.2004.12.012 . hal-00265717

\section{HAL Id: hal-00265717 https://hal.science/hal-00265717}

Submitted on 29 Jan 2020

HAL is a multi-disciplinary open access archive for the deposit and dissemination of scientific research documents, whether they are published or not. The documents may come from teaching and research institutions in France or abroad, or from public or private research centers.
L'archive ouverte pluridisciplinaire HAL, est destinée au dépôt et à la diffusion de documents scientifiques de niveau recherche, publiés ou non, émanant des établissements d'enseignement et de recherche français ou étrangers, des laboratoires publics ou privés. 


\title{
A numerical approach for the study of glass furnace regenerators
}

\author{
Y. Reboussin ${ }^{\text {a }}$, J.F. Fourmigué ${ }^{\mathrm{b}, *}$, Ph. Marty ${ }^{\mathrm{c}}$, O. Citti ${ }^{\mathrm{d}}$ \\ a Saint-Gobain C.R.E.E., 550 Avenue Alphonse Jauffret, B.P. 224, 84306 Cavaillon, France \\ ${ }^{\mathrm{b}}$ C.E.A/GRETh, 17 rue des Martyrs, 38054 Grenoble cedex 9, France \\ ${ }^{c}$ LEGI-GRETh, 17 rue des Martyrs, 38054 Grenoble cedex 9, France \\ d Saint-Gobain Ceramics and Plastics R\&D center, Goddard Road, Northboro, MA 01532, USA
}

Regenerative heat exchangers used in glass industry are complex systems owing to the transient nature of their operating cycle, as well as to the complexity of the heat exchange phenomena involved: aiding mixed convection during the cold period and combined presence of radiation and forced convection during the hot period. The present study describes an open method to simulate the behaviour of such regenerators. Preliminary results allowed us to validate our model in comparison with experimental and/or analytical data obtained on simple geometries. In a second step, a complete validation is proposed on a large scale experimental set-up which reproduces the exact behaviour of an industrial glass furnace regenerator. An original method based on the Boussinesq approximation with a "fictitious thermal expansion coefficient" was successfully introduced for this complete validation.

Keywords: Regenerator; Radiation; Mixed convection; Transient heat transfer

\footnotetext{
${ }^{*}$ Corresponding author. Tel.: +3343878 49 06; fax: +33438785435.

E-mail address: fourmiguejf@chartreuse.cea.fr (J.F. Fourmigué).
} 


\section{Nomenclature}

$c_{p} \quad$ specific heat capacity $(\mathrm{J} / \mathrm{kg} / \mathrm{K})$

$g \quad$ gravity $\left(\mathrm{m} / \mathrm{s}^{2}\right)$

$G r, G r^{*}$ Grashof number

$h \quad$ heat transfer coefficient $\left(\mathrm{W} / \mathrm{m}^{2} / \mathrm{K}\right)$

$L \quad$ characteristic length (m)

$L_{\text {th }} \quad$ thermal loss (\%)

$M \quad$ mass of the regenerator $(\mathrm{kg})$

$\dot{m} \quad$ mass flow rate $(\mathrm{kg} / \mathrm{s})$

$N^{*} \quad$ angular discretization

$p \quad$ porosity

$P^{*} \quad$ pressure $(\mathrm{Pa})$

$P e \quad$ Peclet number

$\mathrm{Pr} \quad$ Prandtl number

$Q \quad$ flow rate $\left(\mathrm{Nm}^{3} / \mathrm{h}\right),\left(\mathrm{O}^{\circ} \mathrm{C}, 1 \mathrm{~atm}\right)$

Re Reynolds number

$R i \quad$ Richardson number

$S \quad$ surface $\left(\mathrm{m}^{2}\right)$

SP suction pyrometer

$T$ temperature (K)

$\mathrm{t}$ time (s)

$U, u \quad$ velocity $(\mathrm{m} / \mathrm{s})$

$u^{*} \quad$ friction velocity $(\mathrm{m} / \mathrm{s})$

$y^{+} \quad$ dimensionless normal distance

Greek symbols

$\beta \quad$ thermal expansion coefficient $(1 / \mathrm{K})$

$\rho \quad$ density $\left(\mathrm{kg} / \mathrm{m}^{3}\right)$

$\varepsilon \quad$ emissivity

$\Phi \quad$ surface heat flux $\left(\mathrm{W} / \mathrm{m}^{2}\right)$

$\Phi^{\mathrm{R}} \quad$ radiant surface heat flux $\left(\mathrm{W} / \mathrm{m}^{2}\right)$

$\eta_{\text {th }} \quad$ thermal efficiency

$\lambda$ conductivity $(\mathrm{W} / \mathrm{m} / \mathrm{K})$

$\Lambda$ reduced length

$\mu \quad$ dynamic viscosity (Pas)

$\Pi \quad$ reduced period

\section{Subscripts}

1/2 half period

b bulk conditions

c cold period 


$\begin{array}{ll}\mathrm{f} & \text { film conditions } \\ \mathrm{h} & \text { hot period } \\ \text { in, out } & \text { inlet, outlet conditions } \\ 0 & \text { reference value } \\ \text { sol } & \text { reference to the solid packing } \\ \text { TF } & \text { turbulent forced } \\ \text { W } & \text { wall conditions }\end{array}$

\section{Introduction}

Regenerators are widely used in many industrial sectors such as cryogenic, metallurgical, chemical process and glass industry. They are indeed attractive due to their wide range of temperature and compactness over which they can be used. A glass industry regenerator for example operates at temperatures up to $1650{ }^{\circ} \mathrm{C}$ whereas a cryogenic regenerator can operate close to a few Kelvin. Although their design depends on the application requirements and can range from rotary regenerators to fixed bed regenerators, regenerator operation relies on the successive alternation of hot and cold periods. During hot periods energy is transferred from the hot fluid to the solid packing, whereas during cold periods the energy previously stored is restored to the cold fluid. A hot period followed by a cold period forms a cycle of operation which duration, for a glass furnace regenerator, is about $40 \mathrm{~min}$. If several cycles of operation are necessary to reach a thermal equilibrium, industrial regenerators usually operate for years under stabilized cyclic conditions and can be considered as fully periodic exchangers.

In the case of a glass regenerator, the solid packing is fixed and basically composed of elementary channels having a square cross-section of about $0.15 \mathrm{~m}$. Hot fluids mainly come from the combustion of the air-fuel mix (exhaust gases) that occurs in the furnace laboratory, whereas the cold fluid is air introduced at the ambient temperature in the regenerator.

During the air period, air is supplied to the regenerator using large fans designed to control the intake flow rate into the regenerator: the flow pattern created by the fan is a forced convection flow. During the exhaust-gas period, the flow rate is controlled through pressure adjustments at the stack.

During the hot period (Fig. 1(a)) the thermal exchange between the fluid and the solid packing is characterized by laminar forced convection combined with radiation [1-3]. This thermal radiation is due to the presence of waste gas which gives rise to a semi-transparent medium. This thermal radiation represents between $80 \%$ and $90 \%$ of the global heat exchange.

On the contrary, during the cold period (Fig. 1(b)), an important temperature gradient occurs between the wall and the core of the air flow which is the origin of free convection flow. This free convection effect produces a flow in the same direction as the forced flow. In that case, the flow is qualified as an aiding mixed convection flow [4-8].

The contribution of free convection to the aiding mixed convection flow decreases from the inlet to the outlet of the channel by a factor of one hundred owing to the increase in temperature. In the same way, the contribution of forced convection decreases by a factor three. These strong variations involve that heat transfer mechanisms significantly evolve from bottom to top. Heat 


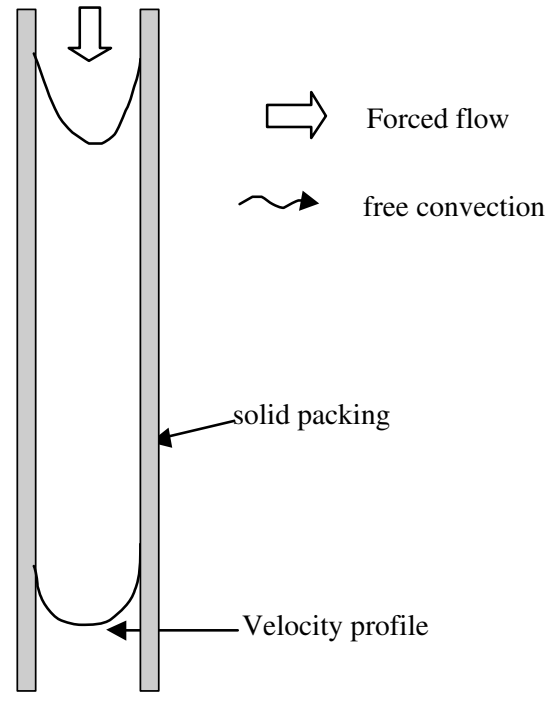

(a)

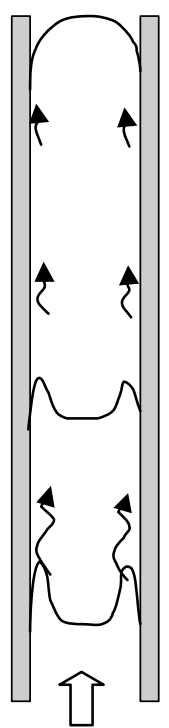

(b)

Fig. 1. Sketch of the flow in the channel of a glass furnace regenerator during each hot (a) and cold (b) period.

exchange is indeed dominated by free turbulent convection mechanisms next to the entrance of the channel whereas, next to the exit, mechanisms of laminar forced convection prevail. Between the entrance and the exit, the flow pattern is then transitional [9].

Eventually it must be noted that since there is no thermal exchange by radiation between the air and the walls of the channel during this period, the mean heat transfer coefficient during the cold period is therefore significantly lower than the one obtained during the hot period. Thus, once the cyclic equilibrium is reached, the air period is considered as the limiting phase for global heat transfer enhancement.

Historically, the study of regenerators has been accompanied by the development of closed methods since the theoretical approach of Hausen [10]. Starting from a theoretical approach, Hausen has introduced two dimensionless numbers, for a fixed bed regenerator: the reduced length $\Lambda=\frac{h S}{m c_{p}}$ and the reduced period $\Pi=\frac{h S}{M c_{p}}\left(t_{1 / 2}-\frac{L}{U}\right)$ from which it is possible to express the thermal efficiency $\eta_{\mathrm{th}}=f\left(\Lambda_{\mathrm{c}}, \Pi_{\mathrm{c}}, \Lambda_{\mathrm{h}}, \Pi_{\mathrm{h}}\right)$. Let us notice that several authors [11-13] have gradually proved the equivalence between this approach and the $\eta_{\mathrm{th}}-\mathrm{NTU}$ approach which is widely used for rotary regenerators. The mathematical equations involved by the problem of the regenerator are quite difficult to solve with closed methods. Many authors have proposed to improve the field of validity, function of the reduced length and the reduced period, as well as the accuracy and the rapidity of the procedures proposed [14-22]. The link between the thermal efficiency, the reduced lengths and the reduced periods is nowadays well known.

Unfortunately, from a practical point of view, the use of such methods not only requires a good knowledge of the operating conditions (intake temperature/flow rates) but also a good knowledge of the heat exchange coefficients during both periods. Because of the complexity of the heat exchange phenomena as well as of the geometry, these approaches are very difficult to implement and very limited application of such models can be achieved. 
The methods used to simulate a regenerator are either closed or open. The closed methods only calculate the cyclic equilibrium and only the periodic conditions are taken into account in the problem. The open methods calculate the successive alternation of hot and cold periods until the cyclic equilibrium is obtained. Owing to the complexity of the phenomena encountered in a glass furnace regenerator, it seems difficult to build a model on the developments made with closed methods and to calculate the mean heat transfer coefficients during both periods by using correlations. That is the reason why the present work proposes an open method, using the Computational Fluid Dynamics (CFD) code Fluent, able to estimate the mean heat transfer coefficients with a satisfactory accuracy. As far as we know, no study concerning the development of an open method to simulate the behaviour of a glass furnace regenerator has been published yet: this is one of the innovations brought by this paper.

This model will be introduced in details and its validation against literature results and experimental data will be extensively presented. CLAIRE large-scale pilot (CEA-GRETh in Grenoble, France) which was used for the acquisition of the experimental data will also be described in this paper.

Eventually, it should be noted that although the geometry of the regenerator tested was close to common industrial designs, the computational work and its validation have been for us a preliminary step prior simulating more complex geometries. As an application of this work, two innovative geometries have indeed been computed. The confrontation of these calculations with experience also showed satisfactory modelling results and enhanced thermal efficiency resulting from the design modifications was revealed. However, none of those results are disclosed in the present document for confidentiality reasons.

\section{Theory}

\subsection{General equations}

For our study, the three-dimensional, time-dependant, momentum equations in the presence of mixed convection combined with radiation are

$$
\begin{aligned}
& \frac{\partial \rho}{\partial t}+\frac{\partial\left(\rho u_{j}\right)}{\partial x_{j}}=0 \\
& \frac{\partial\left(\rho u_{i}\right)}{\partial t}+\frac{\partial}{\partial x_{j}}\left(\rho u_{i} u_{j}\right)=-\frac{\partial P^{*}}{\partial x_{i}}-g \rho+\frac{\partial}{\partial x_{j}}\left(\mu \frac{\partial u_{i}}{\partial x_{j}}\right) \\
& \frac{\partial}{\partial t}\left(\rho c_{p} T\right)+u_{j} \frac{\partial}{\partial x_{j}}\left(\rho c_{p} T\right)=\frac{\partial}{\partial x_{j}}\left(\lambda \frac{\partial T}{\partial x_{j}}\right)+P^{\mathrm{R}}
\end{aligned}
$$

where the radiant volumetric power is defined by the relationship

$$
P^{\mathrm{R}}=-\operatorname{div}\left(\overrightarrow{\Phi^{\mathrm{R}}}\right)=-\int_{0}^{4 \pi} \frac{\partial I}{\partial s} \mathrm{~d} \Omega
$$




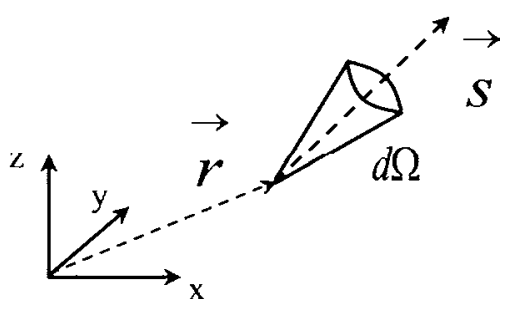

Fig. 2. Definition of the variables used in the radiation calculations.

The directional and monochromatic luminance $I=I(\vec{r}, \vec{s})$ is expressed on an elementary solid angle $\mathrm{d} \Omega$. Its direction is characterized by the unity vector $\vec{s}$ and the coordinates are defined by the vector $\vec{r}$ (Fig. 2).

In this study, we consider an absorbing, emitting and grey medium in which scattering phenomena are negligible. The refraction index is equal to unity, which is an excellent approximation here. Under these assumptions, the radiant transport equation (RTE) can then be written

$$
\vec{s} \cdot \overrightarrow{\operatorname{grad}}(L)=\kappa I^{\mathrm{eq}}-\kappa I
$$

where $I^{\mathrm{eq}}=I^{\mathrm{eq}}(\vec{r})$ is the equilibrium radiant luminance, given by the Planck's function and $\kappa$ the absorption coefficient.

\subsection{Similitude criteria for the cold period}

The dimensionless form of the momentum equation (2) gives rise to two similitude criteria (see Padet $[23,24])$

- The Reynolds number, $R e=\frac{\rho U L}{\mu}$

- The Richardson number, $\quad R i=\frac{\Delta \rho g L}{\rho U^{2}}$

For the cold period, no radiation is taken into account so that the term $P^{\mathrm{R}}$ no longer exists in the energy equation (3). So, its dimensionless form gives one similitude criterion which is

- The Peclet number, $P e=R e \cdot P r$

In this study, the reference length $L$ is the hydraulic diameter $D_{\mathrm{h}}$ of the channel and the velocity scale is the mass-flow velocity $U_{\mathrm{m}}$.

The temperature dependence of the physical parameters $\mu, c_{p}$ and $\lambda$ is considered. Moreover, as the hydraulic diameter is constant, the mass conservation equation (1) involves $\rho U=\rho_{0} U_{0}$. The similitude criteria for the Peclet number and the Reynolds number are satisfied by prescribing a constant velocity $U_{0}$ at the channel entrance. 
Two models can be considered to satisfy the similitude criterion on the Richardson number:

\section{First model}

We express the variation of density as a function of temperature in all the terms of the momentum equation. For a buoyancy-driven flow, this model (called model 1) is known to give poorer results than those given with the use of the Boussinesq approximation.

\section{Second model}

The other possibility (model 2) is based on the Boussinesq approximation. This approximation assumes that (i) the buoyancy term in the momentum equation is written $-g \Delta \rho=\rho_{0} g \beta \Delta T$ and (ii) the density $\rho$ is constant and equal to $\rho_{0}$ in all the other terms. Because of approximation (i), the Richardson number is written

$$
R i=\frac{g \beta \Delta T L}{U^{2}}
$$

and because of approximation (ii), the mass-flow velocity is constant from the bottom to the top of the channel as the equality $\rho U=\rho_{0} U_{0}$ is prescribed by the continuity equation (1). The Richardson number then writes

$$
R i=\frac{g \beta \Delta T L}{U_{0}^{2}}
$$

We notice a difference between Eqs. (9) and (10). As it is necessary to respect the similarity, we propose in this study an original approach by introducing a fictitious thermal expansion coefficient $\beta_{\mathrm{f}}$ instead of the thermal expansion coefficient $\beta$ in Eq. (10)

$$
\beta_{\mathrm{f}}=\beta\left(\frac{\rho}{\rho_{0}}\right)^{2}
$$

where $\rho$ is a function of temperature, $\rho=f(T)$. As the variation of temperature between the entrance and the exit of the channel is of several hundred of degrees, this model considers the thermal expansion coefficient $\beta=\frac{1}{T}$ where $T=T(z)$. The air behaves as a perfect gas so that $\rho T=\rho_{0} T_{0}$ (air inlet conditions), and Eq. (11) can be written

$$
\beta_{\mathrm{f}}=\frac{T_{0}^{2}}{T^{3}}
$$

For this second model, the channel is divided into 40 elementary air volumes. A constant value of the fictitious thermal expansion coefficient is imposed in each one of these volumes.

\subsection{Similitude criteria for the hot period}

During the hot period, the effect of free convection is negligible so that the buoyancy term $-g \rho$ in the momentum equation (2) no longer exists and as a consequence the similitude criterion for the Richardson number.

The Peclet number and the Reynolds number are still two similitude criteria and are respected in the same way as in the cold period. But, the dimensionless energy equation give rise to a new similitude criterion defined as (the refraction index is equal to unity, $n=1$ ) 
- The Planck number, $\frac{\kappa \lambda}{n^{2} \sigma T^{3}}$

where $\sigma$ is the Stefan-Boltzmann constant.

As the dependence of the thermal conductivity with temperature is considered in our models, this similarity criterion will be respected when the absorption coefficient $\kappa$ is correctly taken into account. The weighted sum of grey gas model (WSGGM) is used. First introduced by Hottel and Sarofim [25], it suggests to write the global emissivity as

$$
\varepsilon=\sum_{i} a_{i}\left(1-e^{-\kappa_{i} L}\right)
$$

where $a_{i}$ and $\kappa_{i}$ refer to the grey gas $i$ and denote a weighting term and the absorptivity, respectively. The weighting terms depend on temperature and absorptivity and are function of temperature and characteristic length. In the presence of a gas mixture $\left(\mathrm{H}_{2} \mathrm{O}\right.$ and $\mathrm{CO}_{2}$ in this study), the weighting terms are linked by the relationship $\sum_{i} a_{i}=1$. The coefficients $a_{i}$ and $\kappa_{i} L$ can be explained as those which permit the best estimation of global radiant heat transfer in the gaseous medium owing to its spectral nature. The model WSGG is of great interest thanks to its reduced CPU time and some authors, as Denison and Webb [26], have recently made improvements on it.

\subsection{Calculation procedure}

The calculations need an initial temperature condition in the channel: the value $\left(T_{\mathrm{c}, \text { in }}+T_{\mathrm{h}, \text { in }}\right) / 2$ is then imposed. The transient calculation starts with a hot period followed by a cold period. This operation goes on until the cyclic equilibrium is reached. The convergence criterion is satisfied when the heat regenerated during the hot period is equal to the heat regenerated during the cold period with a difference lower than $1 \%$. For each period, only one step time is made as the evolution of temperature is linear with time and height in the channel [9]. The typical CPU time was approximately 4 weeks on one processor of a modern multiprocessor SUN powerstation.

\section{Test results}

As preliminary work, this section proposes to simplify the problem under consideration by comparing the results of our method to reliable experimental or analytical data from the literature describing each physical phenomenon (free convection, mixed convection, radiation) on a simple geometry (tube, vertical flat plate). Thus, the simulation can be made with a two-dimensional grid, contrary to the complete simulation described in Section 5 which is three-dimensional. For the cold period, a turbulence model using wall functions and a computation grid are chosen by studying both free convection along a vertical plate and aiding mixed convection in a smooth tube. For the hot period, a model of radiation is chosen to describe the RTE.

\subsection{Turbulent free convection}

The well-known $k-\varepsilon$ model combined with wall functions has been developed for forced convection flows. These wall functions have proved their efficiency especially with the introduction of 
low-Reynolds number models for which there exist many different forms. But, as far as we know, very few authors have tested their efficiency to represent free convection. Henkes and Hoogendoorn [27] have compared some of these models for a free convection flow along a vertical flat plate and have proved that their behaviour can be widely different depending on the choice of the wall functions. That is the reason why two types of wall functions, available in Fluent, are tested here in combination with the $k-\varepsilon$ turbulence model: the "standard" wall functions and the "enhanced" wall functions whose form is close to the family of low-Reynolds number models. Grid independence tests have been carried out, but only the results obtained with the most efficient grids are reported in this paper. Thus, we know the range of values in which $y^{+}=\frac{\rho u^{*} y}{\mu}$ at the wall $\left(y_{\mathrm{w}}^{+}\right)$is adequate.

Tsuji and Nagano [28] report very detailed experimental results which are of interest for our problem. The experimental set-up consists of a $4 \mathrm{~m}$ high and $1 \mathrm{~m}$ wide vertical flat plate. These dimensions allow assuming the flow to be two-dimensional. The wall temperature is kept constant to the value $T_{\mathrm{w}}=60^{\circ} \mathrm{C}$ and the bulk temperature of the air is $T_{\mathrm{b}}=16^{\circ} \mathrm{C}$. The physical properties of the air are constant and taken at the film temperature $T_{\mathrm{f}}=\left(T_{\mathrm{w}}+T_{\mathrm{b}}\right) / 2$ (see Table 1$)$.

The two-dimensional calculation field is sufficiently wide $(0.8 \mathrm{~m})$ to neglect the interaction between the lateral boundary and the growing boundary layer along the vertical plate. The grid has 41 nodes in the vertical direction and 43 nodes in the transverse direction.

When the flow is turbulent, that is to say when $G r_{z} P r \geqslant 3.5 \times 10^{9}$, the authors deduce the following correlation from their experimental results

$$
N u_{z}=0.12\left(G r_{z} P r\right)^{1 / 3}
$$

This expression suggests a linear dependence between $N u_{z}$ and the temperature difference between the plate and the external fluid. This statement leads to the conclusion that the heat transfer coefficient is constant. The value of the heat transfer coefficient is reported in Fig. 3 where $z_{\mathrm{cr}}$ corresponds to $G r_{z_{\mathrm{cr}}} \mathrm{Pr}=3.5 \times 10^{9}$.

The results obtained with the "enhanced" wall functions are closer to the correlation than those obtained with the "standard" wall functions ( $h$ becomes a constant after the transition length and the relative error is smaller than with the standard model). Moreover, the local heat transfer coefficient obtained with the "enhanced" wall functions remains constant whereas it slightly increases with the "standard" wall functions. It is interesting to notice that the values of $y_{\mathrm{w}}^{+}$evolve from 15 to 20 along the plate with the "enhanced" wall functions. These values are far from those recommended in forced convection where they should be smaller than the viscous sub-layer (typically $\left.y_{\mathrm{w}}^{+} \approx 1\right)$. In the same way, the velocity profile is also better estimated with the "enhanced" wall functions (Fig. 4).

Indeed, the thickness of the dynamic boundary layer is overestimated by the "standard" wall functions. As for forced convection, the "enhanced" wall functions are then much better than the "standard" wall functions for the determination of both heat exchange and flow pattern.

Table 1

Physical properties of air at film temperature in Ref. [28]

\begin{tabular}{llll}
\hline$\rho\left(\mathrm{kg} \mathrm{m}^{-3}\right)$ & $c_{p}\left(\mathrm{~J} \mathrm{~kg}^{-1} \mathrm{~K}^{-1}\right)$ & $\lambda\left(\mathrm{W} \mathrm{m}^{-1} \mathrm{~K}^{-1}\right)$ & $\mu(\mathrm{Pas})$ \\
\hline 1.12 & 1006 & 0.02718 & $1.901 \times 10^{-5}$ \\
\hline
\end{tabular}




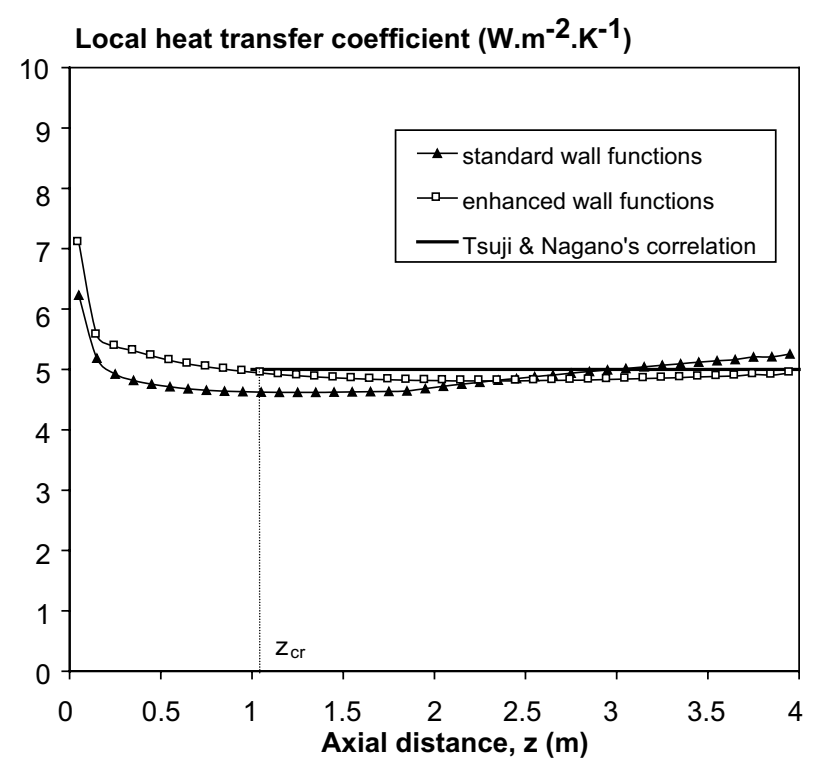

Fig. 3. Evolution of the local heat transfer coefficient along the plate: comparison between our numerical results and Ref. [28].

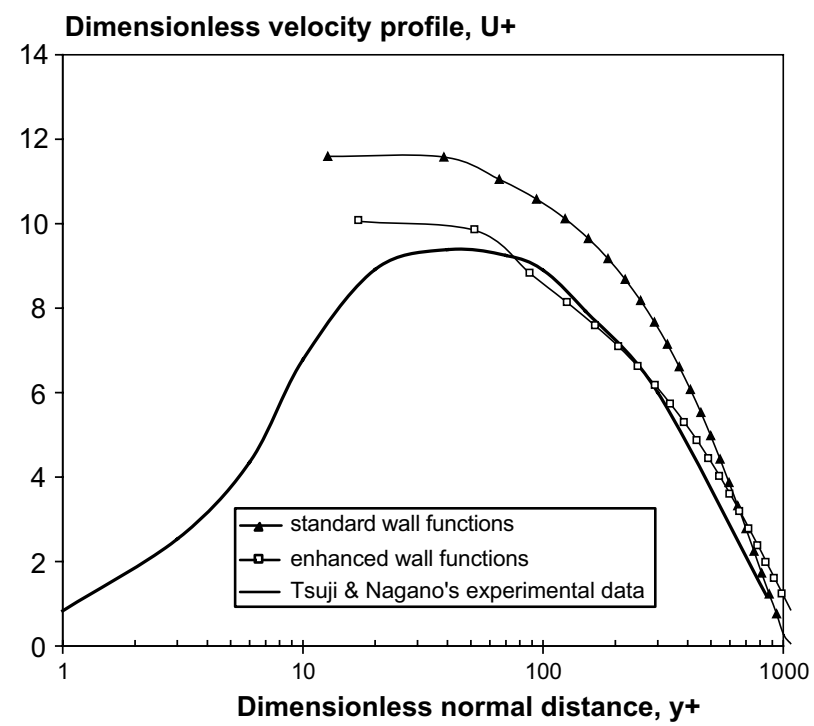

Fig. 4. Evolution of the dimensionless velocity profile as a function of the distance to the wall when $G r_{z}=8.44 \times 10^{10}$ : comparison between our numerical results and Ref. [28].

Yet, contrary to the "standard" wall functions, the "enhanced" wall functions overestimate the temperature difference between the wall and the air: a $20 \%$ difference is observed with respect to the experimental data given in [28] (Fig. 5). 


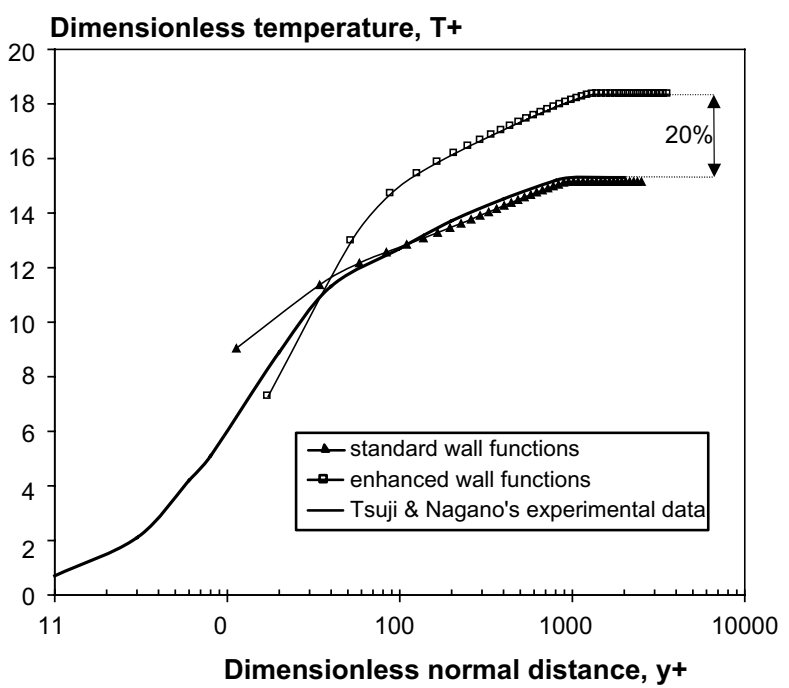

Fig. 5. Evolution of the dimensionless temperature profile as a function of the distance to the wall when $G r_{z}=8.44 \times 10^{10}$ : comparison between our numerical results and Ref. [28].

Nevertheless, for $y^{+} \leqslant 50$, the temperature profile obtained with the "enhanced" wall functions is close to the experimental values. More precisely, the slope of the temperature profile next to the wall is close to the experiments which means that the wall heat flux is well predicted. For $y^{+} \geqslant 50$, the numerical results deviate from the experimental data. Henkes and Hoogendoorn report the same kind of result for different low-Reynolds number models they have tested. The model of Jones and Launder [29] is indeed the best to predict heat transfer for a free turbulent convection flow but it also overestimates the temperature difference between the wall and the air. The explanation can be found by observing the evolution of the turbulent intensity profiles in Fig. 6.

The turbulence model combined with the "enhanced" wall functions underestimates the level of turbulence near the wall. Thus, energy transport from the vicinity of the wall to the core region of the fluid is underestimated. The conclusions made by Henkes and Hoogendoorn are similar concerning the behaviour of the low-Reynolds number model of Jones and Launder.

As it has been highlighted previously, the most important result is that the heat transfer coefficient is well computed. For that reason, the "enhanced" wall functions will be chosen for the rest of this study. This choice is all the more relevant as, during the cold period, the flow pattern is more and more influenced by the mechanisms of forced convection when the air raises up in the channel.

\subsection{Mixed convection}

Many papers reporting experimental studies deal with aiding mixed convection. The geometries studied are vertical flat plates or more often smooth tubes. It is more difficult to find studies which describe a wide range of the mixed convection domain. Even though the amount of data remains weak, we can mention Steiner [30] and Carr et al. [31] for the case of a smooth tube. A recent 


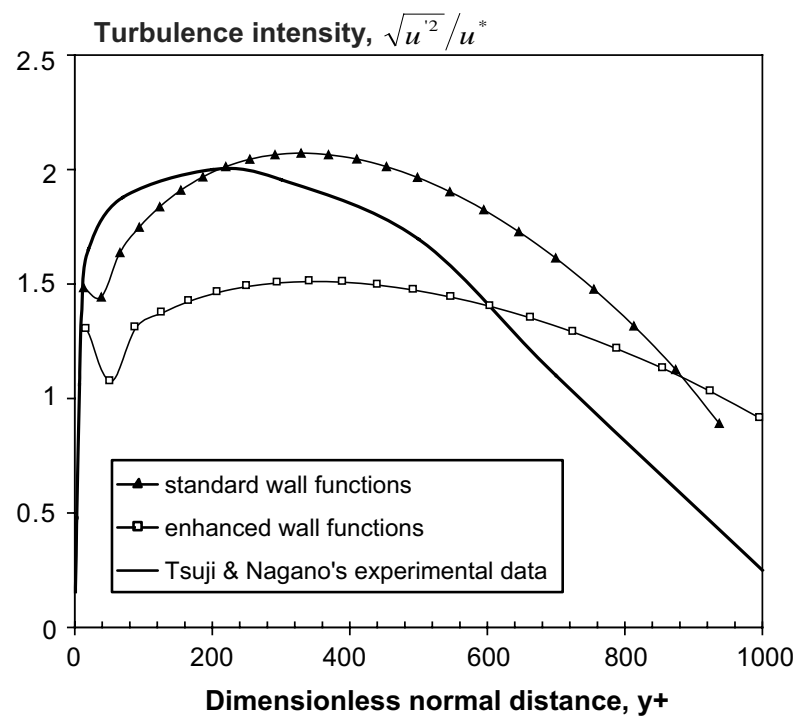

Fig. 6. Evolution of turbulence intensity as a function of the distance to the wall when $G r_{z}=8.44 \times 10^{10}$ : comparison between our numerical results and Ref. [28].

study by Jackson [32] goes further on by carrying out an in-depth experiment. This study will be used in this section for comparison.

Concerning numerical simulations, Cotton and Jackson [33] have tested a low-Reynolds number $k-\varepsilon$ model for a smooth tube in comparison with experimental data. The authors use the lowReynolds number $k-\varepsilon$ model proposed by Launder and Sharma [34] which is a slight modification of the Jones and Launder model. The results of Cotton and Jackson show that the low-Reynolds number $k-\varepsilon$ model is able to predict the aiding mixed convection regime with a good accuracy. They use a fine mesh in their study and the calculation points which are close to the wall are located in the viscous sub-layer $\left(y_{\mathrm{w}}^{+} \approx 0.5\right)$.

To compute low-Reynolds number flows, the Fluent code offers the possibility to use an improved $k-\varepsilon$ model based on the RNG (Re-Normalization Group) theory (see Choudhury [35]). For our problem, the aim of this section is to know if the $k-\varepsilon$ RNG model combined with enhanced wall functions is able to correctly predict the evolution of the heat transfer coefficient in the turbulent aiding mixed convection regime. The mesh used is identical to the one validated for the study with free convection, as we use a $2 \mathrm{D}$-axisymmetric approach. The geometry consists of a $9 \mathrm{~m}$ high tube which diameter is $D=0.0729 \mathrm{~m}$. A distance of $3 \mathrm{~m}$ has been shown necessary to establish the forced turbulent flow. Beyond this zone, the tube is uniformly heated.

The physical properties considered are similar to those used for the study in free convection. The Reynolds number is kept constant at the entrance of the tube and its value is $R e=3000$. The experimental data provided by the authors are introduced by following the evolution of the quantity $\mathrm{Nu} /$ $N u_{\mathrm{TF}}$ as a function of a characteristic dimensionless number, $\mathrm{Gr}^{*} /\left(\operatorname{Re}^{3.425} \operatorname{Pr}^{0.8}\right)$. But, as we are mainly interested in the evolution of the heat transfer coefficient, their results have been expressed in the form $h / h_{\mathrm{TF}}$ where $h_{\mathrm{TF}}$ denotes the heat mean transfer coefficient in the turbulent forced regime (as the thermal conductivity is constant, we can notice that $N u / N u_{\mathrm{TF}}=h / h_{\mathrm{TF}}$ ). 


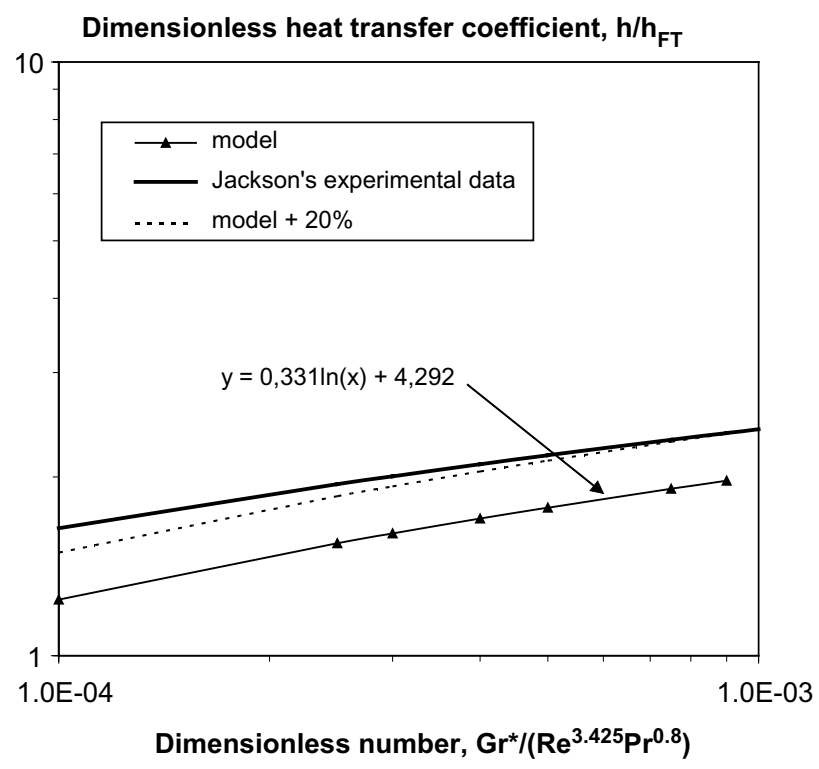

Fig. 7. Axial evolution of the dimensionless heat transfer coefficient: comparison between our numerical results and experimental values from [32].

The local heat transfer coefficient is obtained from the relationship

$$
h=\frac{\Phi}{T_{\mathrm{w}}-T_{\mathrm{b}}}
$$

As already mentioned, the temperature difference between the wall and the fluid is overestimated by $20 \%$ (with enhanced wall functions) what explains the same difference of $20 \%$ between the experimental data from [32] and our model (Fig. 7). Thus, the estimation of heat flux that is exchanged is not affected by this difference. Moreover, the slope of the curve is well computed (close to 1/3). It means that the relative influence of free convection and forced convection is correctly taken into account, with the same mesh used for free convection. It is an important conclusion for the simulation of the cold period of a regenerator.

\subsection{Radiation}

To simulate the thermal radiation exchange, the discrete ordinates (DO) method has been chosen. This method has first been developed for other applications than radiant heat transfer $[36,37]$ and has been extended for thermal radiation. More precisely, the method of Raithby and Chui [38] is used because it is based on a finite-volume method which can be applied with the same computational grids that for fluid flow and convective heat transfer. The discrete ordinates method is well adapted to media with a weak optical thickness as a channel of a regenerator $(\kappa L \cong 0.1)$, and consists of dividing the space into a finite number of solid angles. Using spherical coordinates, the number of solid angles is characterized by the values of $N_{\theta}^{*}$ and $N_{\phi}^{*}$. 
Exact formulations for absorbing, emitting and scattering media in rectangular enclosures have been published [39]. For our test, the dimensions of the geometry are given in Fig. 8.

The first test assumes that the walls are grey with different emissivities $\left(\varepsilon_{\mathrm{w}}=0.4,0.6\right.$ and 0.8$)$ and different wall temperatures imposed to $T_{\mathrm{w}}=273,373$ and $473 \mathrm{~K}$. The gaseous medium is transparent. Moreover, we consider the equality $N_{\theta}^{*}=N_{\phi}^{*}=N^{*}$. The results are given in Table 2.

The good convergence of calculations can be verified on the last line of Table 2. The use of the DO method gives satisfactory results whatever the value of the parameter $N^{*}$. A value of $N^{*}=2$ seems sufficient to simulate radiant heat transfer in a transparent medium.

The second test assumes that the walls of the enclosure have the same properties than in the first test but the gaseous medium is now semi-transparent, isothermal and grey. The results are given in Table 3.

The use of the DO method gives satisfactory results. The values $N^{*}=4$ or 8 give a better result to calculate weak fluxes than the value $N^{*}=2$. On the contrary, the value $N^{*}=2$ is preferable to estimate higher fluxes. Anyway, the relative errors remain negligible whatever may be the value

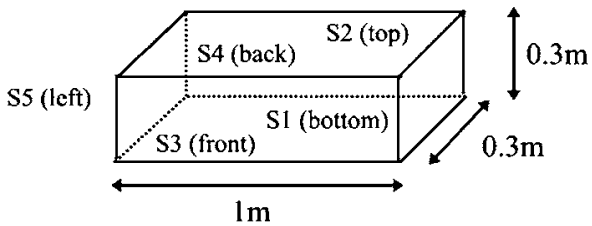

Fig. 8. Dimensions of the enclosure used for testing the radiative model.

Table 2

Results of the test for a transparent medium

\begin{tabular}{lllllll}
\hline Surfaces & $\varepsilon_{\mathrm{w}}$ & $T_{\mathrm{w}}(\mathrm{K})$ & Exact flux $(\mathrm{W})$ & \multicolumn{2}{l}{ Flux $(\mathrm{W})$} & \\
\cline { 5 - 7 } & & & $N^{*}$ & 2 & 4 & 8 \\
\hline 1 or 2 & 0.8 & 273 & -146.3 & -146.56 & -141.84 & -140.68 \\
3 or 4 & 0.4 & 373 & 32 & 32.8 & 30.02 & 29.46 \\
5 or 6 & 0.6 & 473 & 114.3 & 113.76 & 111.82 & 111.22 \\
Sum $(\mathrm{W})$ & & & 0 & 0 & 0 & 0 \\
\hline
\end{tabular}

Table 3

Results of the test for a semi-transparent medium

\begin{tabular}{|c|c|c|c|c|c|c|}
\hline \multirow[t]{2}{*}{ Surfaces } & \multirow[t]{2}{*}{$\varepsilon_{\mathrm{p}}$} & \multirow[t]{2}{*}{$T_{\mathrm{w}}(\mathrm{K})$} & \multirow{2}{*}{$\begin{array}{l}\text { Exact Flux (W) } \\
N^{*}\end{array}$} & \multicolumn{3}{|c|}{ Flux (W) } \\
\hline & & & & 2 & 4 & 8 \\
\hline 1 or 2 & 0.8 & 273 & -345.1 & -343.5 & -335.8 & -334.1 \\
\hline 3 or 4 & 0.4 & 373 & -62.3 & -59.2 & -62.3 & -63 \\
\hline 5 or 6 & 0.6 & 473 & 69.9 & 72.7 & 69.5 & 68.7 \\
\hline \multicolumn{3}{|c|}{ Gaseous medium (W) } & -675 & -660 & -657.2 & -656.8 \\
\hline \multicolumn{3}{|l|}{ Sum (W) } & 0 & 0 & 0 & 0 \\
\hline
\end{tabular}


of $N^{*}$. According to these tests, and as there exists wide temperature gradients in a channel of a regenerator, the value of $N^{*}=4$ is finally chosen, because it is both accurate and acceptable in CPU time.

\section{Experimental set-up and instrumentation}

The work presented below has been done in our laboratory by Lagarenne [9] and co-workers. The test section of the regenerator is presented in Fig. 9: it is limited at the bottom by a low grid and at the top by a high grid.

It consists of a packing of 36 elementary channels, each one made of cruciforms, and having a height equal to $5.040 \mathrm{~m}$. At the base of the channel, the arches support the weight of the packing by means of a transition row. The function of the grids is to ensure that the mass-flow rate is equally distributed among the different channels. In addition, the internal envelop of the test section is insulated to minimize thermal losses. During the hot period, the exhaust gas is coming from a burner $\left(500<Q<2000 \mathrm{Nm}^{3} / \mathrm{h}\right.$ and the temperature can reach $\left.1450^{\circ} \mathrm{C}\right)$ : the flow crosses the regenerator from top to bottom. On the contrary, the air passes through the regenerator from bottom to top during the cold period.

Gas temperature is measured with two suction pyrometers SP1 and SP2. A regular thermocouple placed into a flow would indeed give the equilibrium temperature reached as a result of convective, radiant and conductive heat transfer. On the contrary, the suction pyrometer enables us to minimize the impact of the radiation of the environment and to measure the real gas temperature. The tip of a suction pyrometer is schemed in Fig. 10.

The tip is composed of two anti-radiation shields meant to reduce the effect of environmental radiation [9]. They are made of ceramic materials with low emissivity. But, these shields are not sufficient to ensure a satisfactory representativity of the measurement and gas suction is performed to improve the quality of the measurement. The role of the suction is indeed to increase the convective heat transfer between the thermocouple and the gas so as to reduce the difference between the measured temperature and the real gas temperature. For the present study, the absolute errors are given in Table 4.

During the cold period, the temperature of the air is lower than the temperature of its surroundings. It means that suction cools the thermocouple which is warmed up by radiation. Then, the temperature given by the pyrometer is always above the real gas temperature. That explains the negative errors which are reported in Table 4: absolute error of the suction pyrometers $\left({ }^{\circ} \mathrm{C}\right)$. On the contrary, the real gas temperature is always under-estimated during the hot period. Of course, the higher the temperature is, the more the radiation has a negative impact on the accuracy of the pyrometers. This trend explains the difference between SP1 and SP2 for the cold period. This is also true for the hot period but as the exhaust gas takes part in radiation, which temperature is the real gas temperature, the difference between SP1 and SP2 becomes negligible.

To calculate the mean spatial temperature over the section of the regenerator, we found that it was helpful to maximize the measurement duration or the number, say $N$, of periods. At each new period, the suction pyrometers are displaced and the evolution of gas temperature is recorded as a function of time for a hot and a cold period. A temperature profile can then be obtained at the boundaries of the test section. 


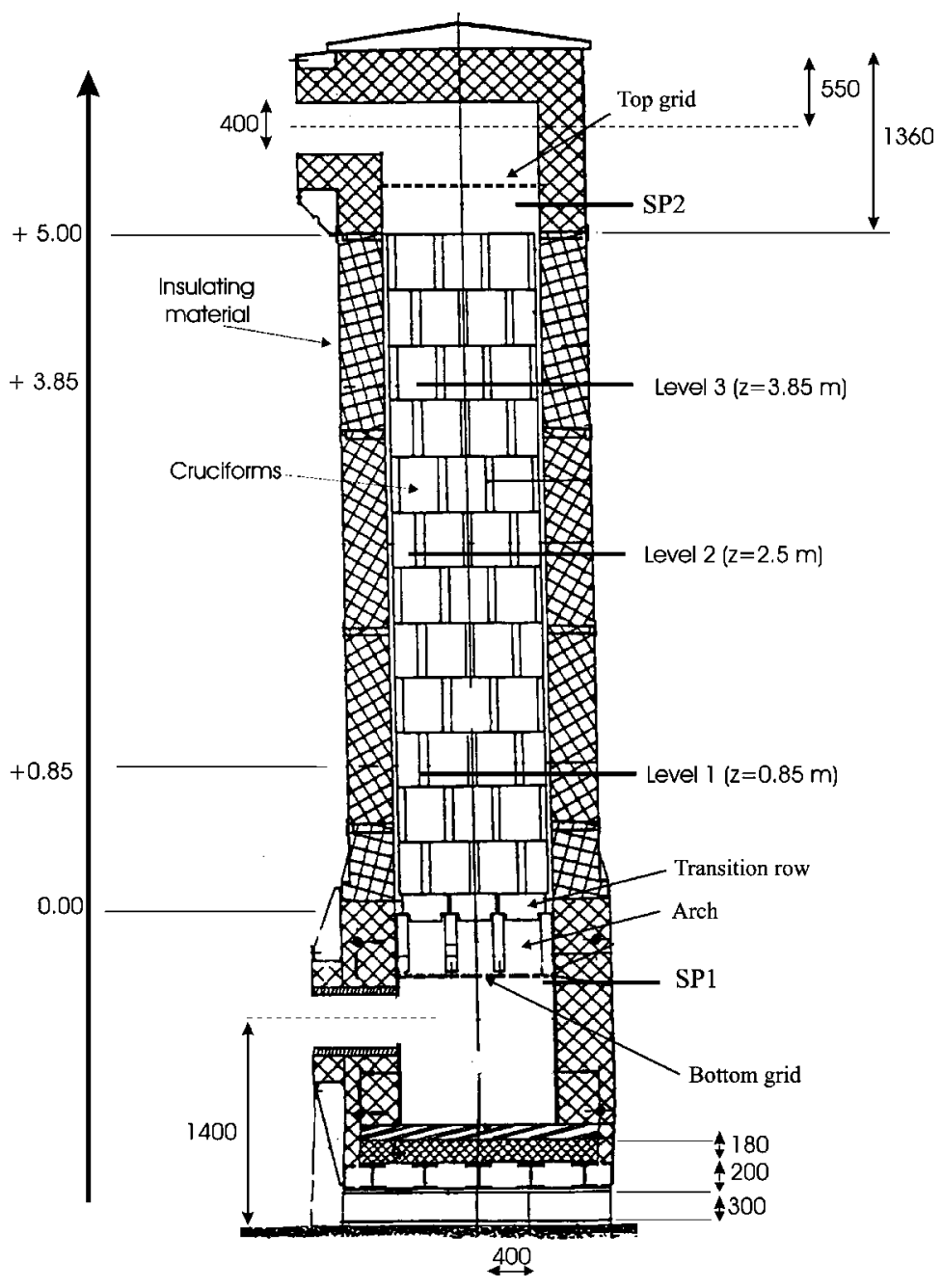

Fig. 9. Test section of our experimental regenerator.

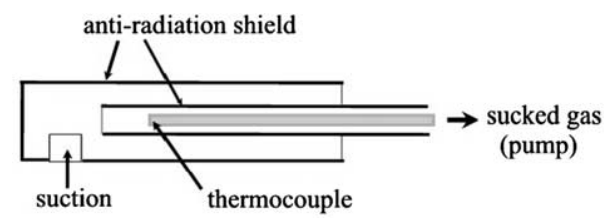

Fig. 10. Tip of one of the suction pyrometers.

The flow rate passing through the regenerator is estimated with a relative error lower than $1 \%$. The distribution of the flow rate in each channel is obtained by dividing the flow rate by the number of elementary channels. In this case and thanks to the distributing grids, the flow rate in an elementary channel is known with a precision of $\pm 3 \%$. 
Table 4

Absolute error of the suction pyrometers $\left({ }^{\circ} \mathrm{C}\right)$

\begin{tabular}{lcc}
\hline Period & SP1 & SP2 \\
\hline Cold & -10 & -25 \\
Hot & +15 & +15 \\
\hline
\end{tabular}

In order to realize local temperature measurements in the walls, it is important to distinguish the peripheral channels from the central channels. Indeed, the peripheral channels undergo the effect of thermal losses through the insulation. It is not the case for the central channels which are considered as insulated channels (Fig. 11).

To realize local measurements, thermocouples were installed into the wings of the cruciforms at several heights in one of these insulated channels. These levels L1, L2 and L3 are visible in Fig. 9. They respectively correspond to the altitude $z=0.85 \mathrm{~m}, z=2.5 \mathrm{~m}$ and $z=3.85 \mathrm{~m}$. At each level, the wing of the instrumented cruciform contains six thermocouples as shown in Fig. 12.

For each level, the spatial mean temperature $T_{\mathrm{m}}$ is defined as the arithmetic average of these 6 thermocouples

$$
T_{\mathrm{m}}=\frac{1}{6} \sum_{i=1}^{6} T_{\mathrm{th}, i}
$$

Lagarenne [9] has experimentally shown that the temperature $T_{\mathrm{m}}$ is dependant of time and that its time-evolution is linear so that $\frac{\partial T_{\mathrm{m}}}{\partial t}=\frac{\Delta T_{\mathrm{m}}}{t_{1 / 2}}$ for both cold and hot periods. The local heat flux regenerated by the cruciforms is then calculated for an elementary channel

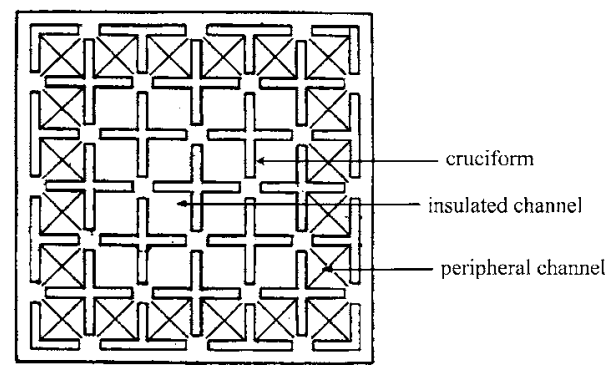

Fig. 11. A-A view of the test regenerator showing the 36 sub-channels.

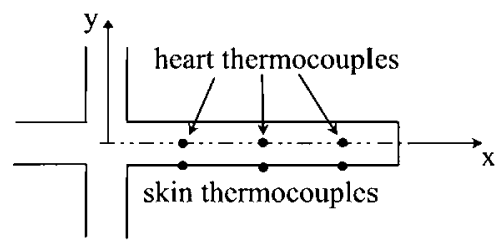

Fig. 12. Distribution of the thermocouples in the wing of a cruciform. 


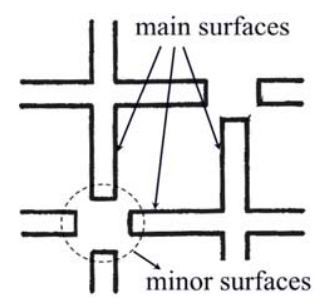

Fig. 13. Scheme of an elementary channel.

Table 5

Comparison of calculation methods for geometric data

\begin{tabular}{lccc}
\hline & $p$ & $S_{\mathrm{c}}\left(\mathrm{m}^{2}\right)$ & $S_{\mathrm{e}}\left(\mathrm{m}^{2}\right)$ \\
\hline Geometric calculation & 0.714 & 0.88 & 106.9 \\
Industrial calculation & 0.701 & 0.817 & 95.7 \\
Difference $(\%)$ & -1.8 & -7.2 & -10.5 \\
\hline
\end{tabular}

Table 6

Working conditions

\begin{tabular}{llllll}
\hline$Q_{\mathrm{c}}\left(\mathrm{Nm}^{3} / \mathrm{h}\right)$ & $Q_{\mathrm{h}}\left(\mathrm{Nm}^{3} / \mathrm{h}\right)$ & $T_{\mathrm{in}, \mathrm{c}}\left({ }^{\circ} \mathrm{C}\right)$ & $T_{\mathrm{in}, \mathrm{h}}\left({ }^{\circ} \mathrm{C}\right)$ & $t_{\mathrm{c}}(\mathrm{s})$ & $t_{\mathrm{h}}(\mathrm{s})$ \\
\hline 800 & 933 & 146 & 1135 & 1260 & 1380 \\
\hline
\end{tabular}

$$
\Phi_{\mathrm{sol}}=\frac{(1-p)}{1000} \rho S_{\mathrm{c}} c_{p} \frac{\partial T_{\mathrm{m}}}{\partial t}
$$

As data acquisition is made for $N$ periods for gas temperature acquisition, the calculation of $T_{\mathrm{m}}$ is reproduced $N$ times. The good consistency of data is then verified. The accuracy of the quantity $\frac{\partial T_{\mathrm{m}}}{\partial t}$ is $\pm 4 \%$. The porosity $p$, the cross section $S_{\mathrm{c}}$ and the surface exchange $S_{\mathrm{e}}$ bring an additional uncertainty. Because of the usual closed design of the packing, it is a current practice in industry to neglect the minor surfaces (in the calculation of the surface exchange $S_{\mathrm{e}}$ ) from which heat exchange is considered as negligible (Fig. 13).

The difference between the two methods is presented in Table 5 .

Finally, for this test, the working conditions are presented in Table 6 . The flow rates are given for 36 channels and the values of $T_{\mathrm{in,c}}$ and $T_{\mathrm{in}, \mathrm{h}}$ are kept constant.

\section{Results and discussion}

The three-dimensional mesh used in this section is a generalisation of the mesh validated in Section 3. It consists of about 25,000 cubic cells. The global results reported in [9] are given in Table 7. The gas temperatures measured by SP1 and SP2 for the hot period $\left(T_{\text {out,h }}\right)$ and the cold period $\left(T_{\text {out, }}\right)$, respectively, were recorded at each half period. 
Table 7

Experimental results of Lagarenne [9] for an elementary channel

\begin{tabular}{lll}
\hline$T_{\text {out }, \mathrm{h}}\left({ }^{\circ} \mathrm{C}\right)$ & $T_{\text {out }, \mathrm{c}}\left({ }^{\circ} \mathrm{C}\right)$ & $\Phi_{\text {sol }}(\mathrm{kW} / \mathrm{m})$ \\
\hline 553 & 852 & 1.3363 \\
\hline
\end{tabular}

Table 8

Comparison of global energy exchanged for an elementary channel (models 1 and 2 have been presented in Section 2)

\begin{tabular}{llcc}
\hline & Experiment & Model 1 & Model 2 \\
\hline$\Phi_{\text {sol }}(\mathrm{kW} / \mathrm{m})$ & 1.3363 & 1.1273 & 1.3107 \\
Difference model/experiment $(\%)$ & & -15.7 & -1.9 \\
\hline
\end{tabular}

The data given in italic undergo the effect of thermal losses whose value is $L_{\mathrm{th}}=\frac{E_{\mathrm{h}}-E_{\mathrm{c}}}{E_{\mathrm{h}}} * 100 \%=15.3 \%$ for this test. The evaluation of the global heat exchange $\Phi_{\text {sol }}$ in the solid packing is made for an elementary channel which is insulated, so it does not undergo the effect of thermal losses. A duration of $1320 \mathrm{~s}$ is considered to calculate the energy $\Phi_{\text {sol }}$, and corresponds to the arithmetic average between $t_{\mathrm{c}}$ and $t_{\mathrm{h}}$.

None of the two models take into account the thermal losses. Concerning global heat exchange, it was then necessary to compare the global heat exchange obtained with the models to the global heat exchange calculated in the solid packing with the help of the experimental results.

The second model is clearly better than the first one which under-estimates the global thermal exchange by more than $15 \%$ (Table 8 ).

This difference between the two models can be understood by following the evolution of the local heat exchange (Fig. 14). If, on account of the experimental uncertainty, the evolution of

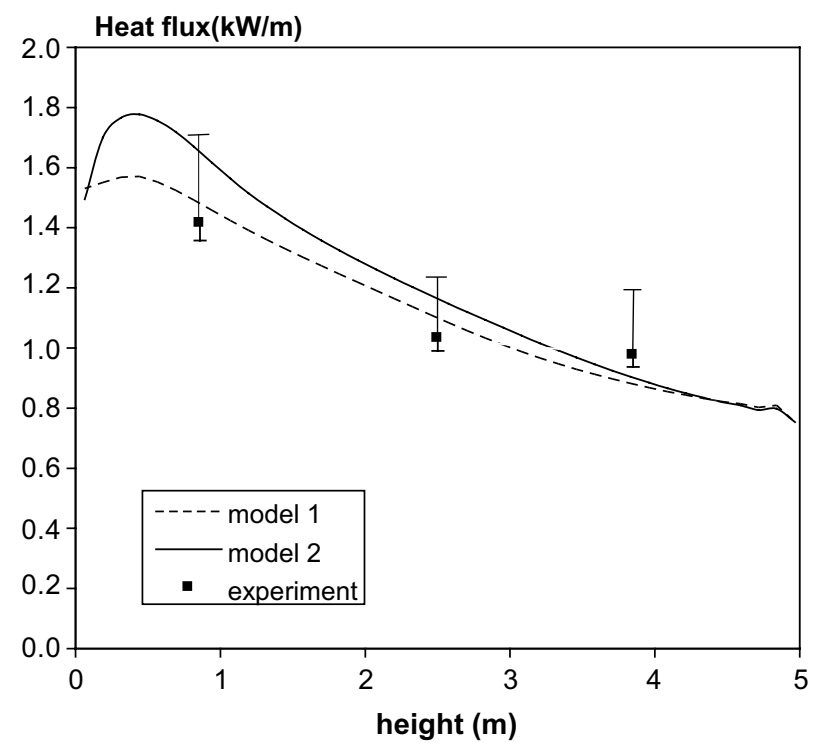

Fig. 14. Profile of the regenerated heat flux for the cold period: comparison between our numerical models and the experimental data from [9]. 


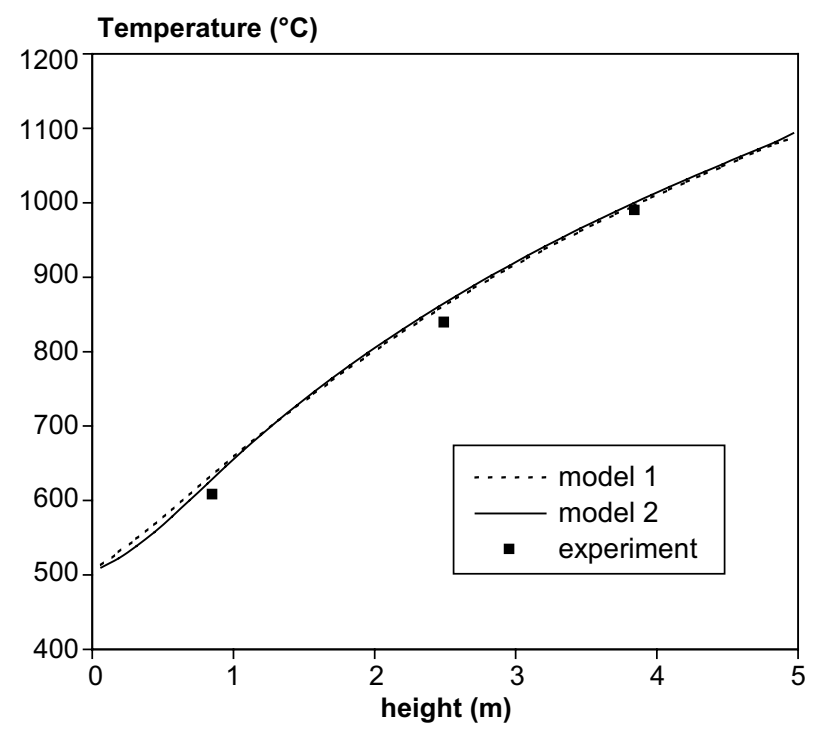

Fig. 15. Wall temperature profile in the middle of the period.

the heat flux regenerated along the channel is well computed by the two models, a discrepancy nevertheless exists at the bottom of the packing (entrance of the air near $z=0$ ).

Indeed, the bottom of the packing is the place where the free convection part of the aiding mixed convection regime is maximum. As the air flows up inside the channel, the influence of the forced convection becomes more and more important and the respective behaviours of the both models get closer and closer. At the top of the channel, where the flow pattern is dominated by the mechanisms of forced convection, the models predict the same amount of heat exchange. This highlights the interest for our fictitious thermal expansion coefficient model (model 2) when calculating the cold period: it combines the advantage of the Boussinesq approximation (good description of free convection) with the respect of the similitude criterion on the Richardson number. Nor the first model, neither the lonely classical Boussinesq approximation can respect these two points.

As the difference with the experimental data does not exceed 4\% (Fig. 15) one can say that the models correctly estimate the axial wall temperature profile. This result confirms that it is not necessary to take into account the spectral nature of radiation to describe radiant heat transfer during the hot period. The weighted sum of grey gas model (WSGGM) is sufficient.

\section{Conclusion}

We have proposed an open method based on a CFD code which has been shown successful. The $k-\varepsilon$ RNG turbulence model combined with the "enhanced" wall functions is chosen to describe the turbulent aiding mixed convection phenomena from the region where heat transfer is dominated by free convection to the region where heat transfer is controlled by forced convection. So, convective heat exchange is correctly computed for the cold period and for the hot period. 
This approach is similar to the family of low-Reynolds number $k-\varepsilon$ models which have proved their efficiency to describe heat transfer in such a flow pattern [27,33].

The DO method is accurate to calculate radiant heat exchange during the hot period. Moreover, as the cold period is the limiting factor of the heat exchanges, the assumption of a grey medium for waste gas is proved to be adequate for a glass furnace regenerator.

An original method is proposed to take into account the effect of the vertical temperature gradient on the evolution of the mixed convection regime. Based on the Boussinesq approximation, it introduces a fictitious thermal expansion coefficient which includes the evolution of density with temperature.

Finally, the model has proved to be of great interest to develop new geometries having an increased thermal efficiency.

\section{Acknowledgement}

The authors gratefully thank Mr Y. Boussant-Roux from the Saint-Gobain Research Center C.R.E.E. for his support.

\section{References}

[1] A. Soufiani, J. Taine, Application of statistical narrow-band model to coupled radiation and convection at high temperature, Int. J. Heat Mass Transfer 30 (3) (1987) 437-447.

[2] A. Soufiani, J. Taine, Experimental and theoretical studies of combined radiant and convective transfer in $\mathrm{CO}_{2}$ and $\mathrm{H}_{2} \mathrm{O}$ laminar flows, Int. J. Heat Mass Transfer 32 (3) (1989) 477-486.

[3] S. Mazumder, F. Modest, Turbulence-radiation interactions in non reactive flow of combustion gases, J. Heat Transfer 121 (1999) 726-729.

[4] M. Nakajima, K. Fukui, Buoyancy effects on turbulent transport in combined free and forced convection between vertical parallel plates, Int. J. Heat Mass Transfer 23 (1980) 1325-1336.

[5] H. Tanaka, S. Maruyama, S. Hatano, Combined forced and natural convection heat transfer for upward flow in a uniformly heated vertical pipe, Int. J. Heat Mass Transfer 30 (1) (1987) 165-174.

[6] J.D. Jackson, M.A. Cotton, B.P. Axcell, Studies of mixed convection in vertical tubes, Int. J. Heat Fluid Flow 10 (1) (1989) 2-15.

[7] T. Aicher, H. Martin, New correlations for mixed turbulent natural and forced convection heat transfer in vertical tubes, Int. J. Heat Mass Transfer 40 (5) (1997) 3617-3626.

[8] R. Celata, et al., Upflow turbulent mixed convection heat transfer in vertical pipes, Int. J. Heat Mass Transfer 41 (1998) 4037-4054.

[9] D. Lagarenne, Récupération d'énergie par les régénérateurs de chaleur des fours de verrerie: Expérimentation, modélisation, optimisation, Thèse INSA/Lyon, 1990.

[10] H. Hausen, Uber die Theorie des Wärmeaustauches in Regeneratoren, Z. Angew. Math. Mech. 9 (1929) $173-200$.

[11] J.E. Coppage, A.L. London, The periodic flow regenerator. A summary of design theory, Trans. ASME J. 75 (7) (1953) 779-787.

[12] R.K. Shah, Thermal design theory for regenerators, in: Heat Exchangers, Thermal Hydraulic Fundamentals and Design, Hemisphere, New York, 1981, pp. 721-763.

[13] P.J. Heggs, Experimental techniques and correlations for heat exchangers surfaces: Regenerators, in: S. Kakaç, R.U. Shah, A.E. Bergles (Eds.), Fundamentals of Low Reynolds Number Forced Convection, Hemisphere, New York, 1982, pp. 369-394.

[14] H. Hausen, Berechnung der Steintemperatur in winderhitzern, Archiv. Für das Eisenhüttenwesen 10 (1939) 473480 . 
[15] A.J. Wilmott, Digital computer simulation of a thermal regenerator, Int. J. Heat Mass Transfer 7 (1964) 12911302.

[16] W.F. Schalkwijk, A simplified regenerator theory, Br. Chem. Eng. 5 (1) (1960) 33-34.

[17] F.W. Schmidt, A.J. Willmott, Thermal Energy Storage and Regeneration, Hemisphere, Mc Graw-Hill, New York, 1981, p. 350.

[18] A. Hill, A.J. Willmott, A robust method for regenerative heat exchanger calculations, Int. J. Heat Mass Transfer 30 (2) (1987) 241-249.

[19] A. Hill, A.J. Willmott, Accurate and rapid thermal regenerator calculations, Int. J. Heat Mass Transfer 32 (3) (1989) 465-476.

[20] B.S. Baclic, G.D. Dragutinovic, Asymmetric-unbalanced counter flow thermal regenerator problem: Solution by the Galerkin method and meaning of dimensionless parameters, Int. J. Heat Mass Transfer 34 (2) (1991) 483-498.

[21] A.J. Willmott, D.P. Knight, Improved collocation methods for thermal regenerator simulations, Int. J. Heat Mass Transfer 36 (6) (1993) 1663-1670.

[22] A.J. Willmott, P.Z. Maguire, Fast Galerkin methods for thermal regenerator modelling, Int. J. Heat Mass Transfer 37 (10) (1994) 1487-1494.

[23] J. Padet, Fluides En écoulement, Méthodes Et Modèles, Masson, Paris, 1991.

[24] J. Padet, Principes Des Transferts Convectifs, Polytechnica, Paris, 1997.

[25] H.C. Hottel, A.F. Sarofim, Radiant Transfer, McGraw-Hill, New York, 1967.

[26] M.K. Denison, R.W. Webb, The spectral line weighted-sum-of-grey-gases model, a review, in: Radiant TransferI, Begell House, New York, 1996, pp. 193-208.

[27] R.A.W. Henkes, C.J. Hoogendoorn, Comparison of turbulence models for the natural convection boundary layer along a heated vertical plate, Int. J. Heat Mass Transfer 32 (1) (1989) 157-169.

[28] T. Tsuji, Y. Nagano, Characteristics of a turbulent natural convection boundary layer along a vertical flat plate, Int. J. Heat Mass Transfer 31 (8) (1988) 1723-1734.

[29] W.P. Jones, B.E. Launder, The prediction of laminarization with a two-equation model of turbulence, Int. J. Heat Mass Transfer 15 (1972) 301-314.

[30] A. Steiner, On the reverse transition of a turbulent flow under the action of buoyancy forces, J. Fluid Mech. 47 (3) (1971) 503-512.

[31] A.D. Carr, M.A. Connor, H.O. Buhr, Velocity, temperature, and turbulence measurements in air pipe flow with combined free and forced convection, J. Heat Transfer (1973).

[32] J.D. Jackson, Influence of buoyancy and thermal boundary conditions on turbulence and effectiveness of cooling by forced or naturally-induced airflow, Conference on the passive cooling of nuclear materials, CEA/Grenoble, France, 27th and 28th October, 1999.

[33] M.A. Cotton, J.D. Jackson, Vertical tube air flows in the turbulent mixed convection regime calculated using a low-Reynolds number $k-\varepsilon$ model, Int J. Heat Mass Transfer 33 (2) (1990) 275-286.

[34] B.E. Launder, B.I. Sharma, Application of the energy-dissipation model of turbulence to the calculation of flow near a spinning disc, Lett. Heat Mass Transfer 1 (1974) 131-138.

[35] D. Choudhury, Introduction to the renormalization group method and turbulence modelling, Fluent Inc., Technical Memorandum TM-107, 1993.

[36] B.G. Carlson, K.D. Lathrop, in: H. Greenspan, C. Kelber, D. Okrent (Eds.), Transport Theory-The Method of Discrete Ordinates in Computing Methods in Reactor Physics, Gordon and Breach, New York, 1968.

[37] K.D. Lathrop, Use of discrete ordinates methods for solution of photon transport problems, Nucl. Sci. Eng. 24 (1966) 381-388.

[38] G.D. Raithby, E.H. Chui, A finite-volume method for predicting a radiant heat transfer in enclosures with participating media, J. Heat Transfer 112 (1990) 415-423.

[39] A.L. Crosbie, R.G. Schrenker, Exact expressions for radiant transfer in a three-dimensional rectangular geometry, J. Quant. Spectrosc. Radiat. Transfer 28 (1982) 507-526. 